\title{
The influence of semantic fit on on-line sentence processing
}

\author{
A. RENÉ SCHMAUDER and MARIE C. EGAN \\ University of South Carolina, Columbia, South Carolina
}

\begin{abstract}
The interactive influence of verb complement preferences and noun phrase semantic fit on resolution of temporary syntactic ambiguity was investigated in an eye movement experiment. The present semantic fit manipulation included noun phrases that fit well as direct objects of the verbs that they followed and noun phrases that were possible but less likely direct objects of the verbs in question. This contrasted with existing research on the use of verb complement preferences and semantic fit during sentence processing, in which processing of noun phrases that are possible direct objects has been compared with processing of noun phrases that are not possible direct objects of the verbs that they follow. Verb complement preference information and noun phrase semantic fit interacted at early stages of on-line sentence processing. Implications of these results for interactive and structural models of sentence processing are discussed.
\end{abstract}

An accurate theory of language comprehension must describe how the human language processor employs different sources of information to resolve the frequent, temporary ambiguities that arise during on-line sentence processing. In the search for an account of how the human sentence processor works, considerable interest has been paid to the role of the verb and to verb subcategorization information (see, e.g., Gorrell, 1991; Holmes, Stowe, \& Cupples, 1989; MacDonald, 1994; MacDonald, Pearlmutter, \& Seidenberg, 1994; Mitchell, 1989; Trueswell \& Tanenhaus, 1994; Trueswell, Tanenhaus, \& Kello, 1993), which specifies which syntactic categories a particular lexical item can take as its complement phrases (Chomsky, 1965, 1981).

Consider the following two sentences:

The woman remembered the flower which the rabbit had eaten.

The woman remembered [that] the flower needed to be watered.

Verbs like remembered can take either a noun phrase (NP) complement, as in (1), or a sentential (S) com-

This research was supported in part by a Carolina Venture Fund Grant to A.R.S. and by a Research and Productive Scholarship Grant to Stanley Dubinsky and A.R.S. from the University of South Carolina. A subset of the research on which this paper is based was presented in a different format at the 36th Annual Meeting of the Psychonomic Society, Los Angeles, November 10-12, 1995. We thank Kathryn Bock, Charles Clifton, Jr., Don Mitchell, Robin Morris, Keith Rayner, and anonymous reviewers for helpful comments on an earlier version of the paper and Terri Fergusson, Julia Richardson, Kendra Robertson, and Angie Slice for their assistance in data collection. Correspondence concerning this article should be addressed to A. R. Schmauder, Department of Psychology, University of South Carolina, Columbia, SC 29208 (e-mail: rene@jethawk.psych.sc.edu). plement, as in (2). Through the double-underlined NP, sentences like (1) and (2) (without the optional that) are potentially ambiguous, because the NP the flower may be taken either to be the direct object (DO) of the verb, as in (1), or to be the subject NP of a following S complement, as in (2) without the optional complementizer that. The single-underlined phrase in (1) disambiguates toward the S-complement reading when the that is absent. If verbs' complement preferences are stored and can be used immediately, the sentence processor can anticipate the most likely complements and avoid the DO/S-complement ambiguity.

To make use of potential benefits to sentence processing provided by lexical information, many current interactive models of sentence processing assume that lexical information is richly articulated and influences all stages of sentence processing (Ford, Bresnan, \& Kaplan, 1982; Holmes et al., 1989; MacDonald, 1994; MacDonald et al., 1994; Marslen-Wilson \& Tyler, 1987; McClelland, 1987; McClelland, St. John, \& Taraban, 1989; Pearlmutter, Daugherty, MacDonald, \& Seidenberg, 1993; Trueswell, 1996; Trueswell \& Tanenhaus, 1994; Trueswell, Tanenhaus, \& Garnsey, 1994; Trueswell et al., 1993; Tyler, 1989; Tyler \& Marslen-Wilson, 1977). In contrast, structural models, such as the garden path model, posit a primary role during parsing of $X$-bar rules and a small set of sentence processing principles such as minimal attachment and late closure (Frazier, 1979, 1987, 1989; Frazier \& Fodor, 1978; Frazier \& Rayner, 1982; Kimball, 1973; see Mitchell, 1994, and Tanenhaus \& Trueswell, 1995, for reviews of structural and interactive models). ${ }^{1}$ Two assumptions distinguish structural models from interactive models: the structural guidance assumption, that the first structural analysis at a point of ambiguity is dictated by grammatical rules and structural processing principles; and the single-structure assumption, that multiple alterna- 
tive syntactic analyses are not considered in parallel. Rather, a single structural representation of the sentence is constructed and considered as each new word is attached to the existing structure.

Verb subcategorization information may be available immediately to the processing system when a verb is read or heard. Quick use of verb information is compatible with the structural guidance assumption, if verb information influences the degree of commitment to or the ease of revision of the initial structural attachment constructed by the parser (Frazier, 1994; Inoue \& Fodor, 1994). Also, lexical information may be used in quick, incremental assessment of the plausibility of an initial, on-line structure constructed by the parser. This too is compatible with structural guidance, as long as the syntactic structure dictated by the grammar and structural processing principles is built first (see, e.g., Speer \& Clifton, 1998). Since early use of lexical information is compatible with both interactive and structural models, we chose to focus on the single-structure assumption in the research reported here. Evidence that multiple structures are considered in parallel cannot be accommodated by the garden path model without modification.

Examining processing of sentences like the temporarily ambiguous (2) without the complementizer that and the unambiguous version of (2), researchers have reported results consistent with interactive models (Holmes, 1987; Holmes, Kennedy, \& Murray, 1987; Mitchell \& Holmes, 1985; Trueswell et al., 1993) and with structural models (Ferreira \& Henderson, 1990; Frazier \& Rayner, 1982; Holmes et al., 1987; Rayner \& Frazier, 1987). Attempting to sort out the mixed results, researchers have focused on joint influences of verb preference and plausibility of a potential DO on processing of the DO/S-complement ambiguity (Garnsey, Pearlmutter, Myers, \& Lotocky, 1997; Holmes et al., 1989, Trueswell et al., 1993). For instance, Garnsey et al. collected eye movement data from subjects reading sentences with a $\mathrm{DO} / \mathrm{S}$-complement ambiguity. They manipulated verb preference and the semantic fit of the critical ambiguous NP. In first-pass reading times from the temporarily ambiguous NP region, Garnsey et al. found an NP $\times$ complementizer interaction following only verbs that preferred a DO complement (NP-preference verbs). The NP fit $\times$ complementizer interaction was not reliable after verbs that preferred a sentential complement (S-preference verbs). This interaction reflected a missingcomplementizer effect after NP-preference verbs for NPs that were not good DOs; there was no effect of the missing complementizer for NPs that were good DOs. Garnsey et al. also found a main effect of the missing complementizer in first-pass data from the critical NP region following both NP-preference verbs and S-preference verbs. In the disambiguating verb phrase region, they observed missing-complementizer effects in sentences with NPpreference verbs but not in sentences with S-preference verbs. Also, NP fit influenced processing in the disambiguating region only for NP-preference verbs. The lack of a reliable NP fit $X$ complementizer interaction in the disambiguating region led Garnsey et al, to conclude that readers were garden pathed after NP-preference verbs even if the critical NP was not a good DO. Further, they noted that $\mathrm{S}$-preference verbs were followed by no garden path effect in the disambiguating region. Since garden pathing was not observed after S-preference verbs, Garnsey et al. concluded in favor of an interactive processing model rather than the garden path model.

Examination of the literature revealed the following fact, which may account for at least some of the conflicting findings on processing of the temporary $\mathrm{DO} / \mathrm{S}$-complement ambiguity. Researchers examining processing of $\mathrm{DO} / \mathrm{S}-$ complement ambiguous sentences (e.g., Garnsey et al., 1997; Holmes et al., 1989; Trueswell et al., 1993) have reasonably begun such investigations by testing strong semantic and syntactic manipulations to see whether the processor is sensitive to these manipulations. They included sentences in which the critical NPs could not possibly be DOs of the verbs that they followed on the transitive reading of those verbs. For instance, in Holmes et al.'s (1989) materials, more S-preference verbs (73\%) than NP-preference verbs (37\%) were followed by implausible NPs that could not possibly serve as DOs, as in The inspector realized the vehicle. Trueswell et al. (1993) noted that Ferreira and Henderson's (1990) materials included NPs that could not be DOs of some of their NPpreference verbs. Yet in Trueswell et al.'s (1993) materials, nearly all S-preference verbs did not permit or facilitate a DO continuation. These items were not DO/S-complement ambiguous through the critical NP region because the DO interpretation was not possible. Garnsey et al. also included a number of items in which the critical NP could not possibly be the DO of the preceding verb (e.g., The executive concluded the bank.).

Two types of violation result if the parser considers a DO analysis of an NP that is not a possible DO of the preceding verb: (1) Semantic anomaly results when the system notes that the NP in question does not fit semantically as a DO; and (2) syntactic anomaly results if the parser attempts to attach an NP as the DO of a verb that cannot take an NP complement. Exactly this attachment violation is predicted by the garden path structural model. The type and distribution of such semantic and syntactic anomalies within material sets may have influenced whether the existing data supported a structural parsing model or an interactive model. Also, given that some research has found an early effect on sentence processing of strong semantic fit manipulations (e.g., Garnsey et al., 1997; Trueswell et al., 1993), it is important to explore whether these interesting findings would extend to a manipulation in which semantic fit differences exist but items do not give rise to semantic or syntactic anomaly.

To address these theoretical issues, in the present experiment we further investigated the role of semantic fit and verb complement preferences in sentence processing of S-complement sentences. But we did not want to assess behavior of the language processing system in the presence of semantic and syntactic anomalies. Rather, and critically, we used NPs that varied in plausibility as DO NPs without being semantically anomalous. This exact 
manipulation was necessary so that we could investigate the impact on sentence processing of fluctuations in plausibility like those presumably present in normal language use. Further, we ensured that all of our DO/S-complement ambiguous materials actually were temporarily ambiguous at the critical NPs.

To these ends, we employed a plausibility manipulation where either the better fit or the neutral fit NP was a possible $\mathrm{DO}$ of the preceding verb on the transitive reading, but the better fit NP was a more likely DO NP than was the neutral fit NP. We determined DO plausibility in a norming study with each NP-preference and each Spreference verb and the critical noun phrase that followed it in the experimental materials. There were no semantic violations in our materials, and plausibility of NPs as DOs was evaluated with respect to each verb to ensure a fit between that verb and the following NP. This avoided possible differences in NP fit across verbs that might be generated by using the same critical NP with both an NPpreference verb and an S-preference verb as in Trueswell et al. (1993; Garnsey et al., 1997, and Kennison, 1995, also noted this point). Furthermore, all NP-preference verbs and all S-preference verbs in our experiment permitted either a DO or an S complement, so structural attachment of the critical NP was potentially ambiguous in all sentences.

We examined how the semantic fit manipulation, verb complement preference information, and temporary syntactic ambiguity simultaneously influenced fixation times during silent reading, to explore different operating stages of the sentence processing system (Rayner, Sereno, Morris, Schmauder, \& Clifton, 1989). Sample materials are listed in Table 1.

Interactive accounts predicted that any (or all) available source(s) of information, including semantic fit information as well as structural preferences, might influence sentence processing at a given point of interest. Critically, interactive accounts allow that multiple structural analyses might be active, perhaps to varying degrees, at a given point during parsing. In contrast, structural models assume serial analysis of structure, prohibiting simultaneous construction of multiple structural analyses. Evidence that only the DO analysis of the temporarily ambiguous NP is considered would support the single-

\section{Table 1}

Sample Materials

\footnotetext{
NP-Preference verbs

Better fit NP, no that [that present]:

The photographer observed [that] the scene was not very attractive.

Neutral fit NP, no that [that present]:

The photographer observed [that] the radio was not very attractive.

S-Preference verbs

Better fit NP, no that [that present]

The suspect admitted [that] the robbery was not a good idea.

Neutral fit NP, no that [that present]:

The suspect admitted [that] the quarrel was not a good idea.

Note-Double underline, ambiguous region. Single underline, disambiguating region.
}

structure assumption of structural models, whereas evidence that both the DO and the S-complement structure were available at the temporarily ambiguous NP would support interactive models.

\section{METHOD}

\section{Participants}

Thirty-two native English speakers from the University of South Carolina with normal, uncorrected vision received credit as part of the Psychology Department's human participant pool for their participation in the main experiment.

\section{Materials and Design}

Sixteen NP-preference verbs and 16 S-preference verbs were selected in a norming study. Participants were given a proper name followed by a verb and asked to complete this sentence fragment. The NP-preference and S-preference verbs were placed in S-complement sentence sets like those in Table $1 .^{2}$ Two sentence frames were written per verb, manipulating fit of the postverbal NPs as potential DOs of a matrix verb. Better fit postverbal NPs were tempting DO NPs; neutral fit postverbal NPs were less plausible DOs; but neutral fit NPs were not anomalous as DOs. For instance, a photographer is more likely to observe a scene than a radio, although observing a radio is not semantically anomalous (see Table 1).

\section{Norming Study}

A norming study established that the participant population shared the experimenters' intuitions about semantic fit of the critical postverbal NPs. For each item set, the critical better fit and neutral fit NPs were placed in simple, transitive sentences, as in the following example

\section{a. The photographer observed the scene.}

b. The photographer observed the radio.

In the norming study, 56 participants received human participant pool credit for rating the resulting sentences to establish whether sentences with better fit NPs were rated as more realistic than sentences with neutral fit NPs. The sentences were split into four different booklets, each with 32 experimental sentences and equal numbers of better fit and neutral fit NPs, so that each verb appeared only once in a booklet. Thus, of the 56 participants, 14 rated the sentences in one of each of the four booklets, which also contained filler sentences that varied in plausibility. The sentence order in each booklet was randomized. A 6-point rating scale, adapted from Taraban and McClelland (1988), was present at the top of each page with the range of numerical values presented below each sentence: 5 , extremely realistic sentence, 4 , very realistic; 3 , moderately realistic; 2 , somewhat realistic; 1 , barely realistic; 0 , not at all realistic. A practice set of six example sentences, representing each of the six possible rating values, was reviewed prior to the rating task.

The mean rating assigned to items with better fit NPs was 3.42; the mean rating assigned to items with neutral fit NPs was 2.73 , a reliable difference $[t(63)=6.62, p<.0001]$. The participants analysis was also reliable $[t(55)=8.20, p<.0001]$. Sentences with S-preference verbs had a mean rating of 3.28 with better fit NPs, versus a mean rating of 2.63 with neutral fit NPs. Sentences with NP-preference verbs had a mean rating of 3.56 with better fit NPs and a mean rating of 2.83 with neutral fit subject NPs. Verb preference did not interact with NP fit reliably $(F<1)$, nor did it have a significant main effect $\left[F(1,62)=1.97, M S_{\mathrm{e}}=0.909, p>.1\right]$. The norming study supported the experimenters' intuitive judgments about NP semantic fit.

\section{Main Experiment}

Verb preference (NP preference vs. S preference), critical NP fit (better fit as DO NP vs. neutral fit as DO NP), and complementizer 
presence (no that vs. that present) were manipulated within participants. Sentences with the same verb were presented in two different blocks, counterbalanced across participants. Materials were rotated in a Latin square design within blocks, so participants saw an equal number of the four sentence types for each level of verb preference (better fit no that, better fit that present, neutral fit no that neutral fit that present). Head nouns in the critical NPs were matched within two characters in length and also on frequency of use (Francis \& Kučera, 1982). Critical NPs were followed by at least three words, all of which fit on the first line of the 72 character-per-line sentence display.

\section{Apparatus and Procedure}

A Fourward Technologies Dual Purkinje Image eye movement monitoring system was interfaced with an IBM PS/2 Model 80 computer to record eye movements from participants' right eyes. Viewing was binocular; four characters equaled $1^{\circ}$ of visual angle. A bite plate minimized head movements. Participants were instructed to read normally for comprehension. Display brightness was adjusted to a comfortable level, the equipment was calibrated, and a block of 15 practice trials was presented. Participants then read two blocks of 64 sentences, with a brief break between blocks. Thirty-two of the sentences in each block were experimental sentences, and the remaining items were filler sentences that differed across blocks. ${ }^{3}$ Participants received feedback on comprehension question errors. The session took approximately $45 \mathrm{~min}$

\section{RESULTS}

All participants performed at better than $90 \%$ accuracy on comprehension questions.

The measures of fixation time reported here are firstpass reading time and measures of rereading or reprocessing, including second-pass fixation time in a region and percentage of refixations of a region. The first-pass reading time measure is sensitive to initial stages of word and sentence processing, reflecting at least lexical access and activation; the rereading measures reflect sentence integration and reanalysis processes (Rayner et al., 1989). The first-pass times were calculated by summing all fixations in a region prior to the reader's leaving that region either to fixate later words or to regress to a prior word. The second-pass fixation times were calculated as the average refixation times from a region; when a region was not refixated, zero refixation time was averaged into the second-pass measure reported here. Results are presented for the analyses by participants $\left(F_{1}\right)$ and by items $\left(F_{2}\right)$ where reliable. Verb type was a between-items factor in the items analyses.

The scoring regions of interest were (1) the temporarily ambiguous subject NP of the embedded sentence complement (NP region), and (2) the next three words in the embedded verb phrase, which disambiguated the critical $\mathrm{NP}$ as subject of the sentence complement (D3 region). Both regions included short function words which sometimes were not fixated, so we did not examine fixations on individual words in the regions (Rayner et al., 1989).

In the no-that conditions, the critical postverbal NPs were temporarily ambiguous: In the absence of additional information, the NP was ambiguous as either a DO of the matrix verb or the subject of an embedded S complement. Interactive models predicted effects of NP fit and verb preference in first-pass reading time data from the NP and D3 regions, assuming that those sources of information were available at lexical access (McElree \& Griffith, 1995). Furthermore, interactive models predicted that both the DO analysis and the S-complement analysis would be considered at the ambiguous region, whereas the single-structure assumption of structural models predicted that we would observe evidence that only the DO analysis was considered.

\section{Initial Sentence Processing}

First-pass reading times: Disambiguating region. Mean first-pass data from the disambiguating D3 region are shown in Table 2. First-pass reading times in the D3 region indicated that the complementizer effect was reliable by participants, with a mean first-pass time of $608 \mathrm{msec}$ for no-that conditions and $586 \mathrm{msec}$ for thatpresent conditions $\left[F_{1}(1,31)=5.84, M S_{\mathrm{e}}=10,726, p<\right.$ $\left..02 ; F_{2}(1,30)=2.09, M S_{\mathrm{e}}=17,118, p>.1\right]$. The data showed that participants detected the missing complementizer on the NP following both NP-preference and S-preference verbs. Ferreira and Henderson (1990) also reported this result (without a semantic manipulation) and argued in support of the garden path model. However, we

Table 2

Mean First-Pass Times (and Standard Deviations) in Milliseconds for the Regions of Interest

\begin{tabular}{|c|c|c|c|c|c|c|c|c|c|c|c|c|}
\hline \multirow[b]{3}{*}{ Condition } & \multicolumn{6}{|c|}{ Noun Phrase-Preference Verbs } & \multicolumn{6}{|c|}{ Sentence Complement-Preference Verbs } \\
\hline & \multicolumn{2}{|c|}{ No That } & \multicolumn{2}{|c|}{ That Present } & \multirow[b]{2}{*}{$\Delta$} & \multirow[b]{2}{*}{$M$} & \multicolumn{2}{|c|}{ No That } & \multicolumn{2}{|c|}{ That Present } & \multirow[b]{2}{*}{$\Delta$} & \multirow[b]{2}{*}{$M$} \\
\hline & $M$ & $S D$ & $M$ & $S D$ & & & $M$ & $S D$ & $M$ & $S D$ & & \\
\hline \multicolumn{13}{|c|}{ NP Subject of the Embedded S Complement (NP Region) } \\
\hline Better fit NP & 394 & 128 & 402 & 131 & -8 & 398 & 459 & 138 & 390 & 207 & +69 & 424 \\
\hline Neutral fit NP & 435 & 162 & 383 & 117 & +52 & 409 & 450 & 155 & 397 & 127 & +53 & 423 \\
\hline$\Delta$ & -41 & & +19 & & & & +9 & & -7 & & & \\
\hline$M$ & 414 & & 392 & & +22 & & 454 & & 393 & & +61 & \\
\hline \multicolumn{13}{|c|}{ First Three Words in Embedded Verb Phrase (D3 Region) } \\
\hline Better fit NP & 642 & 175 & 587 & 210 & +55 & 614 & 632 & 235 & 573 & 164 & +59 & 602 \\
\hline Neutral fit NP & 574 & 182 & 587 & 183 & -13 & 580 & 582 & 197 & 595 & 169 & -13 & 588 \\
\hline$\Delta$ & +68 & & 0 & & & & +50 & & -22 & & & \\
\hline$M$ & 608 & & 587 & & +21 & & 607 & & 584 & & +23 & \\
\hline
\end{tabular}


report data below that do not support their account. We observed that complementizer absence interacted with NP fit in first-pass data from the $\mathrm{D} 3$ region $\left[F_{1}(1,31)=6.95\right.$, $M S_{\mathrm{e}}=22,645, p<.01 ; F_{2}(1,30)=4.86, M S_{\mathrm{e}}=15,080$, $p<.04]$. First-pass times were long for the no-that, better fit condition, a $57-\mathrm{msec}$ effect, but not for the neutral fit condition (13 msec in the opposite direction); the effect was present and of similar magnitude after both NPpreference and S-preference verbs. Our results show that semantic fit of the critical NP evoked or exaggerated the temporary syntactic misanalysis of those NPs as DOs; the misanalysis was detected in the D3 region, which was inconsistent with the DO analysis. These results will be discussed further in the next section and in the Discussion.

Finding a reliable NP fit interaction with these materials is notable because the NP fit manipulation did not yield semantic or syntactic anomaly, and because the fit manipulation was representative of the shades of plausibility that we presume to be present in normal language use. The fact that the fit effects were detected in firstpass reading time data provides important support for the sensitivity of the sentence processor.

First-pass reading times: Temporarily ambiguous NP region. First-pass data from the NP region (see Table 2) showed a large missing complementizer effect, $435 \mathrm{msec}$ for no that versus $393 \mathrm{msec}$ for that present $\left[F_{1}(1,31)=6.85, M S_{\mathrm{e}}=32,572, p<.02 ; F_{2}(1,30)=\right.$ $\left.12.76, M S_{\mathrm{e}}=8,434, p<.001\right]$. This effect was due to three conditions: the neutral fit, NP-preference verb condition and both S-preference verb conditions. There was a missing-complementizer effect for both better fit and neutral fit NPs after S-preference verbs (cf. Trueswell et al., 1993). The interaction between verb type and complementizer absence was marginal $\left[F_{1}(1,31)=3.49\right.$, $M S_{\mathrm{e}}=13,506, p>.07 ; F_{2}(1,30)=3.03, M S_{\mathrm{e}}=8,434$, $p>$.09]. Simple effects tests showed a main effect of complementizer absence for the S-preference verbs $\left[F_{1}(1,31)=9.61, M S_{\mathrm{e}}=24,723, p<.004 ; F_{2}(1,15)=\right.$ 21.16, $\left.M S_{\mathrm{e}}=5,627, p<.001\right]$, but not for NP-preference verbs $\left[F_{1}(1,31)=1.52, M S_{\mathrm{e}}=21,355, p>.2 ; F_{2}(1,15)=\right.$ $\left.1.26, M S_{\mathrm{e}}=11,240, p>.2\right]$. However, in the first-pass data from the NP region, there also was a 52-msec complementizer effect for neutral fit NPs following NP-preference verbs, and no complementizer effect when better fit NPs followed NP-preference verbs. The no-that/thatpresent contrast was marginal for neutral fit NPs after NP-preference verbs $\left[F_{1}(1,31)=2.95, M S_{\mathrm{e}}=30,129\right.$, $\left.p<.1 ; F_{2}(1,15)=3.29, M S_{\mathrm{e}}=119,252, p=.09\right]$.

Better fit NPs following NP-preference verbs were interpreted as DOs. In contrast, when readers encountered neutral fit NPs, one of two things evidently occurred. The DO analysis of the neutral fit NPs may have been constructed, with quick assessment of plausibility leading to longer reading times on neutral fit NPs in the nothat condition. We doubt this fast plausibility assessment account, because there were no semantic violations when neutral fit NPs were interpreted as DOs. Rather, we believe that both the DO analysis of the neutral fit NPs and the S-complement analysis were considered in parallel, even after NP-preference verbs. Parallel consideration of two structures may have slowed processing because neither analysis received enough support to "win" over the other structure. A fast plausibility assessment account predicted residual processing difficulty in the D3 region, where it was clear that the neutral fit NP was not the DO of the verb, in line with predictions of the garden path model. However, there was no complementizer effect in the D3 region for neutral fit NPs after NP-preference verbs. The multiple-analyses alternative predicted that the processing load should be resolved at the embedded verb phrase, which forced selection of the S-complement analysis. This was observed: Processing difficulty was evident on the NP region for no-that, neutral fit NPs after NP-preference verbs, but we found no processing difficulty in this condition in the $\mathrm{D} 3$ region. We will discuss this result further in the Discussion.

\section{Sentence Reanalysis}

Second-pass processing times: Disambiguating region. Mean second-pass fixation times are reported in Table 3 . The only salient pattern in the mean second-pass fixation times from the disambiguating D3 region was

Table 3

Mean Second-Pass Fixation Times (and Standard Deviations) in Milliseconds for the Regions of Interest

\begin{tabular}{|c|c|c|c|c|c|c|c|c|c|c|c|c|}
\hline \multirow[b]{3}{*}{ Condition } & \multicolumn{6}{|c|}{ Noun Phrase-Preference Verbs } & \multicolumn{6}{|c|}{ Sentence Complement-Preference Verbs } \\
\hline & \multicolumn{2}{|c|}{ No That } & \multicolumn{2}{|c|}{ That Present } & \multirow[b]{2}{*}{$\Delta$} & \multirow[b]{2}{*}{$M$} & \multicolumn{2}{|c|}{ No That } & \multicolumn{2}{|c|}{ That Present } & \multirow[b]{2}{*}{$\Delta$} & \multirow[b]{2}{*}{$M$} \\
\hline & $M$ & $S D$ & $\bar{M}$ & $S D$ & & & $M$ & $\overline{S D}$ & $M$ & $\overline{S D}$ & & \\
\hline \multicolumn{13}{|c|}{ NP Subject of the Embedded S Complement (NP Region) } \\
\hline Better fit NP & 197 & 185 & 129 & 132 & +68 & 163 & 100 & 122 & 88 & 110 & +12 & 94 \\
\hline Neutral fit NP & 245 & 225 & 155 & 166 & +90 & 200 & 174 & 171 & 183 & 204 & -9 & 178 \\
\hline$\Delta$ & -48 & & -26 & & & & -74 & & -95 & & & \\
\hline$M$ & 221 & & 142 & & +79 & & 137 & & 135 & & +2 & \\
\hline \multicolumn{13}{|c|}{ First Three Words in Embedded Verb Phrase (D3 Region) } \\
\hline Better fit NP & 223 & 269 & 165 & 206 & +58 & 194 & 171 & 222 & 168 & 184 & +3 & 170 \\
\hline Neutral fit NP & 220 & 229 & 188 & 218 & +32 & 204 & 161 & 169 & 199 & 231 & -38 & 180 \\
\hline$\Delta$ & +3 & & -23 & & & & +10 & & -31 & & & \\
\hline$M$ & 221 & & 176 & & +45 & & 166 & & 184 & & -18 & \\
\hline
\end{tabular}


an interaction between verb preference and complementizer presence $\left[F_{1}(1,31)=6.03, M S_{\mathrm{e}}=20,921, p<.02\right.$; $\left.F_{2}(1,30)=3.79, M S_{\mathrm{e}}=16,183, p=.06\right]$. After NPpreference verbs, both better fit and neutral fit NPs were evidently considered as possible DOs as the sentence was fixated during reprocessing. This was evident as a missing-complementizer effect in second pass times in the D3 region. This pattern was not present for sentences with S-preference verbs. After NP-preference verbs, better fit NPs led to a somewhat larger garden path effect in secondpass times on the disambiguating D3 region, but no interactions with NP fit were reliable in the second-pass data.

Finding garden path evidence in the D3 region in second-pass data for the NP-preference verb, neutral fit NP conditions contrasted with the first-pass effects in the D3 region, where there was little evidence of garden pathing in the NP-preference verb, neutral fit NP condition. Taken together, the results show that there was delayed consideration of the DO interpretation of the neutral fit NPs after NP-preference verbs. The garden path model's single-structure assumption predicted that the DO analysis should have been considered immediately rather than at this later stage of processing. These results indicated that verb preference information influenced reanalysis and sentence integration processes in addition to having an influence on initial processing stages.

Second-pass processing times: Temporarily ambiguous NP region. Second-pass times from the NP region showed that NP-preference verbs were refixated for more time than were S-preference verbs [182 vs. $136 \mathrm{msec}$; $F_{1}(1,31)=20.42, M S_{\mathrm{e}}=13,080, p<.0001 ; F_{2}(1,30)=$ $\left.4.69, M S_{\mathrm{e}}=27,956, p<.04\right]$. There was also a main effect of complementizer absence, with no-that conditions refixated for $170 \mathrm{msec}$ and that-present conditions for $129 \operatorname{msec}\left[F_{1}(1,31)=16.36, M S_{\mathrm{e}}=12,764, p<.0003\right.$; $\left.F_{2}(1,30)=5.7, M S_{\mathrm{e}}=18,789, p<.03\right]$. Verb preference interacted with complementizer presence $\left[F_{1}(1,31)=\right.$ $17.62, M S_{\mathrm{e}}=11,213, p<.0002 ; F_{2}(1,30)=5.03, M S_{\mathrm{e}}=$ $18,789, p<.04]$. After NP-preference verbs, there was a 79-msec missing-complementizer effect in the secondpass data. There was no reliable missing-complementizer effect on the NP region for the $S$-preference verb conditions in second-pass data. Evidently, the S-preference verbs' argument structure information assisted recovery from the missing-complementizer effects in the first-pass data.

NP fit influenced second-pass times on the NP region, with shorter second-pass time on better fit NPs, $128 \mathrm{msec}$, than on neutral fit NPs, 189 msec $\left[F_{1}(1,31)=41.64\right.$, $M S_{\mathrm{e}}=11,340, p<.0001 ; F_{2}(1,30)=25.17, M S_{\mathrm{e}}=8,993$, $p<.0001]$. This showed that NP fit influenced sentence integration processes as well as affecting garden pathing in the first-pass data. Furthermore, in the second-pass data, NP fit interacted with verb preference in the NP region $\left[F_{1}(1,31)=6.16, M S_{\mathrm{e}}=11,827, p<.02 ; F_{2}(1,30)=\right.$ $\left.4.38, M S_{\mathrm{e}}=8,993, p<.05\right]$. Following NP-preference verbs, mean second-pass time was $37 \mathrm{msec}$ shorter for better fit NPs than for neutral fit NPs; after S-preference verbs, mean second-pass time was $84 \mathrm{msec}$ shorter for better fit NPs than for neutral fit NPs. The interaction reflected the brief second-pass time in the S-preference better fit condition ( $94 \mathrm{msec})$. In the D3 region following NP-preference verbs, better fit NP items were refixated for more time in the no-that condition than in the thatpresent condition, a pattern not present in the first-pass data from the NP region. The pattern of results in secondpass data for neutral fit NPs after NP-preference verbs was similar to the first-pass pattern. The pattern of saccades leaving the D3 region to refixate earlier regions of the sentence (regressions) supported the second-pass data. The rereading data supported the claim that the temporarily ambiguous NP was interpreted as the DO of the matrix verb more often when both verb preference and the NP's lexical semantics supported the DO interpretation. Also, taken together, measures of reprocessing indicated that verbs' complement preferences aided readers in reaching a final sentence interpretation.

\section{DISCUSSION}

In the present experiment, we explored whether a more subtle NP semantic fit manipulation than typically is used influenced processing of sentences with a DO/Scomplement temporary structural ambiguity. We avoided outright subcategorization and semantic violations by ensuring that the temporarily ambiguous NPs were possible sentence continuations on either the DO or the sentential complement interpretation, prior to the disambiguating region. NP fit influenced the processing reflected in first-pass reading times, with NP fit effects present on the critical NP region itself.

Observing early NP fit effects with these materials is remarkable for at least two reasons: First, the NP fit manipulation that we employed did not resolve a syntactic ambiguity; and second, the fit manipulation was within the range of semantic plausibility and thus did not invoke any processing associated with infelicity or ungrammaticality. Regarding the first point, Trueswell et al. (1994) demonstrated early semantic fit effects with an animacy manipulation in reduced relative clauses. But their inanimate condition (e.g., The evidence examined ...) ruled out the active verb reading of their reduced relative clauses, and thus there was no syntactic ambiguity in their inanimate condition. In our sentences, both DO and S-complement interpretations were possible at the NP, even in the neutral fit conditions. Thus our NP fit effects extend existing research on lexical-semantic manipulations to cases in which syntax truly was ambiguous. Regarding the second point, our results show that the sentence processing system is quite sensitive, since it detected an early, yet pronounced, influence of the NP fit manipulation on structural processing.

The garden path model predicted that regardless of whether verbs preferred NP or S-complements, temporarily ambiguous NPs would initially be interpreted as DOs. Further, the garden path model predicted that NP fit would influence only the revision of this initial DO 
analysis. This prediction was supported by data only from the NP-preference verb, better fit NP condition, in which both verb information and fit information converged on a single interpretation. There, the classic garden path effect was observed, with no processing difficulty in the ambiguous condition on the critical NP but difficulty in the disambiguating region. Difficulty in the disambiguating region reflected revision of an initial DO attachment, which also led to increased second-pass reading times on regions of interest.

The other three conditions revealed a pattern of data at odds with the garden path model's predictions. Following NP-preference verbs, first-pass reading times were long on ambiguous neutral fit NPs. Because these NPs were not bad or infelicitous DOs, we do not support a plausibility assessment explanation, according to which a garden path model would construct DO structure and quickly evaluate and then reject implausible DOs. Rather, it seems that both the DO and S-complement analyses were active to some degree, with the DO analysis more active following NP-preference verbs. The multiple active interpretations and the neutral fit of the NP then led to competition and reordering of the syntactic interpretations. We know that this reordering was resolved on the NP and resulted in the S-complement interpretation's being selected, for in the NP-preference verb, neutral fit condition, no difficulty was present in first-pass times from the syntactically disambiguating D3 region. However, the processor was sensitive to the conflict between the verb subcategorization and semantic fit information, for there was inflated second-pass time on the NP region in the ambiguous neutral fit condition, as the processor confirmed that despite the NP-complement verb preference, the Scomplement construction was accurate.

In the S-preference verb, ambiguous better fit NP condition, we also observed the effects of a reordering of structural interpretations. Evidently verb information activated the S-complement analysis, but the DO analysis was also active to some degree. When a better fit (as DO) NP was encountered next, long first-pass times in the ambiguous condition reflected reordering of structural interpretations, with the DO analysis competing with, or becoming more active than, the S-complement analysis. This competition resulted in long first-pass times on the disambiguating D3 region. The S-complement analysis then was selected during first-pass reading of the D3 region in the S-preference, better fit condition; no further processing difficulty was present in the ambiguous condition in second-pass reading times.

Further, reordering of structural interpretations occurred in the S-preference, neutral fit condition. Again, the verb made the $\mathrm{S}$-complement analysis most active, but the DO analysis was also active. Since the neutral fit NP could serve as a DO, there was competition between the two structural interpretations, with longer first-pass time in the ambiguous condition. Here, neutral fit of the NP as DO led to quick resolution of this structural competition, on the NP region. There was no first-pass processing difficulty in the D3 region, indicating that the S-complement interpretation was selected earlier. The garden path model cannot account for the evidence of competition between structural preferences and NP fit that we observed in this experiment.

Consider a possible alternative account of our results. The probabilistic single-analysis model discussed by Mitchell and colleagues (Mitchell, 1994; Mitchell \& Cuetos, 1991) allows that in structures like the one that we examined, sometimes the S-complement structure is built rather than $\mathrm{DO}$ structure. According to probabilistic models, if one or the other single structure is built with different probabilities, across a data set such as ours, it can appear that both structures were constructed in parallel. We note, though, that significant variability in which of the two possible structures is constructed suggests that the processing system "knows" that there are two possible structural interpretations. And if the processor "knows" that there are two possible structures, whether in fact two complete syntactic structures are constructed is a secondary question.

Of further interest is the fact that our results differed from those of Garnsey et al. (1997) in certain ways. We observed a missing-complementizer effect in the NP region after S-preference verbs as well as after NP-preference verbs. This disparity reflects a difference in the sentence processor's operation in our study as compared with Garnsey et al.'s study, where many poor fit NPs were anomalous on a transitive reading of the matrix verb. Also, in the disambiguating region, Garnsey et al. observed a reliable missing-complementizer effect only for NP-preference verb conditions, but we observed it for both verb complement preference types. With the present materials, the DO interpretation of the better fit NPs was considered after both NP-preference and S-preference verbs because the DO interpretation was possible; both the NP's lexical semantics and verbs' subcategorization information permitted the DO continuation. In this way, our data extend Garnsey et al.'s work.

Several sources of lexical information can indicate to the processor that more than one structure is possible at a point of structural ambiguity. For instance, in cases of lexical category ambiguity, lexical category information requires that two different structures be built to represent both interpretations of the category-ambiguous word (for evidence of multiple access, see Folk \& Morris, 1995; Onifer \& Swinney, 1981; Seidenberg, Tanenhaus, Leiman, \& Bienkowski, 1982). Also, if an ambiguous phrase is compatible with more than one argument structure in a verb's lexical representation, more than one syntactic structure will be needed to represent both readings. At least in this second case, the fact that there are two readings requires that multiple relations between the available argument structures and the critical phrase be represented explicitly in the system. These representations may be syntactic structures or some other hierarchical representation capturing the association between the relevant lexical elements.

One might ask, what does it mean for a structure to be "available"? Single-analysis models posit that only one 
possible syntactic structure is constructed at a time; in these models, a structure is not available until it is built at the level of syntax. By contrast, in MacDonald's constraintbased model (MacDonald, 1994; MacDonald et al., 1994), verb argument structures may be activated partially as a function of frequency of use, and plausibility information may also influence activation levels. If active argument structure information entails the construction of multiple syntactic structures, then MacDonald's model violates the single-structure assumption assumed by standard structural parsing models. But MacDonald (1994) noted that perhaps "complete phrase markers need not aiways be built" (p. 161, note 2); if this suggestion is correct, the single-structure issue is moot. However, MacDonald's suggestion does not solve the problem of how the processing system represents the different hierarchical relations between arguments that are often entailed by multiple interpretations. That is, our results were consistent with both the DO analysis and the S-complement analysis of the ambiguous NP region being available to the processor. Either multiple syntactic structures were built, or multiple representations were created at a nonsyntactic, nonlexical level of representation, a level of representation that can represent hierarchical relations between arguments. These results emphasize the need for further research investigating how lexical information is projected to a syntactic or other nonlexical level of representation.

The present results are incompatible with the garden path model or with any other model which states that only one structure is built regardless of verb preferences. We have argued further that multiple representations encoding the available structural possibilities are present in the system, even if only one of those representations ends up with a full representation at the syntactic level of representation.

\section{REFERENCES}

CHomsky, N. (1965). Aspects of a theory of syntax. Cambridge, MA: MIT Press.

CHOMSKY, N. (1981). Lectures on government and binding (5th ed.). Dordrecht: Foris.

Ferreira, F., \& Henderson, J. M. (1990). Use of verb information in syntactic parsing: Evidence from eye movements and word-by-word self-paced reading. Journal of Experimental Psychology: Learning, Memory, \& Cognition, 16, 555-568.

FOLK, J., \& MORRIS, R. K. (1995, November). Semantic ambiguity across syntactic category in reading. Poster presented at the 36 th Annual Meeting of the Psychonomic Society.

Ford, M., Bresnan, J. W., \& Kaplan, R. M. (1982). A competencebased theory of syntactic closure. In J. W. Bresnan (Ed.), The mental representation of grammatical relations (pp. 727-796). Cambridge, MA: MIT Press

Francis, W. N., \& Kučera, H. (1982). Frequency analysis of English usage: Lexicon and grammar. Boston: Houghton Mifflin.

FRAZIER, L. (1979). On comprehending sentences: Syntactic parsing strategies. Bloomington, IN: Indiana University Linguistics Club.

FraZIER, L. (1987). Sentence processing: A tutorial review. In M. Coltheart (Ed.), Attention and performance XII: The psychology of reading (pp. 559-586). Hillsdale, NJ: Erlbaum.

Frazier, L. (1989). Against lexical generation of syntax. In W. D. Marslen-Wilson (Ed.), Lexical representation and process (pp. 505528). Cambridge. MA: MIT Press.
Frazier, L. (1994). Sentence (re)-analysis. Paper presented at the 7th annual CUNY Sentence Processing Conference, New York.

FraZIER, L., \& FodOR, J. D. (1978). The sausage machine: A new twostage parsing model. Cognition, 6, 291-325.

Frazier, L., \& RaYNer, K. (1982). Making and correcting errors during sentence comprehension: Eye movements in the analysis of structurally ambiguous sentences. Cognitive Psychology, 14, 178-210.

Garnsey, S. M., Pearlmutter, N. J., Myers, E., \& Lotocky, M. A. (1997). The contributions of verb bias and plausibility to the comprehension of temporarily ambiguous sentences. Journal of Memory \& Language, 37, 58-93

GORRELL, P. (1991). Subcategorization and sentence processing. In R. C. Berwick, S. P. Abney, \& C. Tenny (Eds.), Principle-based parsing (pp. 279-300). Dordrecht: Kluwer.

HOLMES, V. M. (1987). Syntactic parsing: In search of the garden path. In M. Coltheart (Ed.), Attention and performance XII: The psychology of reading (pp. 587-599). Hillsdale, NJ: Erlbaum.

Holmes, V. M., Kennedy, A., \& Murray, W. S. (1987). Syntactic structure and the garden path. Quarterly Journal of Experimental Psychology, 39A, 277-292.

Holmes, V. M., STOWE, L., \& CuPples, L. (1989). Lexical expectations in parsing complement-verb sentences. Journal of Memory \& Language, 28, 668-689.

INOUE, A, \& FoDOR, J. (1994). Information-paced parsing of Japanese. In R. Mazuka \& N. Nagai (Eds.), Japanese sentence processing (pp. 9-63). Hillsdale, NJ: Erlbaum.

KENNISON, S. M. (1995). The role of verb-specific lexical information in syntactic ambiguity resolution. Unpublished doctoral dissertation, University of Massachusetts, Amherst.

KimBALL, J. (1973). Seven principles of surface structure parsing in natural language Cognition, 2, 15-47.

MacDonald, M. C. (1994). Probabilistic constraints and syntactic ambiguity resolution. Language \& Cognitive Processes, 9, 157-201.

MacDonald, M. C., Pearlmutter, N. J., \& Seidenberg, M. S. (1994). On the lexical nature of syntactic ambiguity resolution. Psychological Review, 101, 676-703.

MarsLen-Wilson, W. D., \& TyleR, L. K. (1987). Against modularity. In J. Garfield (Ed.), Modularity in knowledge representation and natural language understanding (pp. 37-62). Cambridge, MA: MIT Press.

McClelland, J. L. (1987). The case for interactionism in language processing. In M. Coltheart (Ed.), Attention and performance XII: The psychology of reading (pp. 3-36). Hillsdale, NJ: Erlbaum.

McClelland, J. L., St. John, M., \& Taraban, R. (1989). Sentence comprehension: A parallel distributed processing approach. Language \& Cognitive Processes, 4, 287-338.

MCElreE, B., \& GrIfFITH, T. (1995). Syntactic and thematic processing in sentence comprehension: Evidence for a temporal dissociation. Journal of Experimental Psychology: Learning, Memory, \& Cognition, 21, 134-157.

Mitchell, D. C. (1989). Verb-guidance and other lexical effects in parsing. Language \& Cognitive Processes, 4, 123-154.

MitchelL, D. C. (1994). Sentence parsing. In M. A. Gernsbacher(Ed.), Handbook of psycholinguistics (pp. 375-409). San Diego: Academic Press.

Mitchell, D. C., \& Cuetos, F. (1991). The origins of parsing strategies. In C. Smith (Ed.), Current issues in natural language processing (pp. 1-12). Austin: University of Texas, Center for Cognitive Science.

Mitchell, D. C., \& Holmes, V. M. (1985). The role of specific information about the verb in parsing sentences with local structural ambiguity. Journal of Memory \& Language, 24, 542-559.

ONIFER, W., \& SWINNEY, D. A. (1981). Accessing lexical ambiguities during sentence comprehension: Effects of frequency of meaning and contextual bias. Memory \& Cognition, 9, 225-236.

Pearlmutter, N. J., Daugherty, K., MacDonald, M. C., \& Seiden$\mathrm{BERG}, \mathrm{M} . \mathrm{S}$. (1993). Constraint satisfaction in main verb/reduced relative ambiguities. Paper presented at the 6th annual CUNY Sentence Processing Conference, Amherst, MA.

Rayner, K., \& Frazier, L. (1987). Parsing temporarily ambiguous complements. Quarterly Journal of Experimental Psychology, 39A, $657-673$ 
Rayner, K., Sereno, S. C., Morris, R. K., Schmauder, A. R., \& CLIFTON, C.. JR. (1989). Eye movements and on-line language comprehension processes. Language \& Cognitive Processes, 4, 21 -49.

Seidenberg, M. S., Tanenhaus, M. K., Leiman, J. M., \& BienkowSKI, M. (1982). Automatic access of the meanings of ambiguous words in context: Some limitations of knowledge-based processing Cognitive Psychology, 14, 489-537.

SPEER, S. R., \& Clifron, C., JR. (1998). Plausibility and argument structure in sentence comprehension. Memory \& Cognition, 26, $965-978$

Tanenhaus, M. K., \& Trueswell, J. C. (1995). Sentence comprehension. In J. L. Miller \& P. D. Eimas (Eds.), Speech, language, and communication (pp. 217-262). San Diego: Academic Press.

TARABAN, R., \& MCClELl.AND. J. L. (1988), Constituent attachment and thematic role assignment in sentence processing: Influences of contentbased expectations. Journal of Memory \& Language, 27, 597-632.

TRUESWELL, J. C. (1996). The role of lexical frequency in syntactic ambiguity resolution. Journal of Memory \& Language, 35, 566-585.

Trueswell, J. C., \& Tanenhaus, M. K. (1994). Toward a lexicalist framework of constraint-based syntactic ambiguity resolution. In C. Clifton, Jr., L. Frazier, \& K. Rayner (Eds.), Perspectives on sentence processing (pp. 155-180). Hillsdale, NJ: Erlbaum.

Trueswell, J. C., Tanenhaus, M. K., \& Garnsey, S. M. (1994). Semantic influences on parsing: Use of thematic role information in syntactic ambiguity resolution. Journal of Memory \& Language, 33, 285-318.

Trueswell, J. C., Tanenhaus, M. K., \& Kello, C. (1993). Verb-specific constraints in sentence processing: Separating effects of lexical pref- erence from garden-paths. Journal of Experimental Psychology: Learning, Memory, \& Cognition, 19, 528-553.

TYLER, L. K. (1989). The role of lexical representations in language comprehension. In W. D. Marslen-Wilson (Ed.), Lexical representation and process (pp. 439-462). Cambridge, MA: MIT Press.

TYLER, L. K., \& MARSLEN-WiLson, W. D. (1977). The on-line effects of semantic context on syntactic processing. Journal of Verbal Learning \& Verbal Behavior, 16, 683-692.

\section{NOTES}

1. The minimal attachment principle leads the parser to build what can be considered to be the simplest possible syntactic structure at any given point during parsing. See Frazier (1979) for a detailed account.

2. A complete set of materials is available from the authors upon request.

3. Note that $50 \%$ of the sentences contained S complements. Trueswell et al. (1993) criticized Ferreira and Henderson (1990) for a high ratio of $S$ complements to non-S complements in their materials, which may have increased expectation for S-complement structure. However, this worked against the hypotheses that we were testing: If participants anticipated S-complement structure, we would not expect to find evidence that participants pursued a DO analysis of the ambiguous NP. As presented below, evidence of garden pathing was observed despite the proportion of $S$ complements in the materials. Furthermore, the singlestructure assumption predicts that a single syntactic analysis will be constructed regardless of participants' expectations.

(Manuscript received March 25, 1997;

revision accepted for publication July 25,1997 .) 